\title{
Translation, Cross-Cultural Adaptation, and Psychometric Properties of the Malay-Translated Kihon Checklist Among Elderly Patients In The Emergency Department
}

Suhaila Naema Laili Suhairi

Universiti Kebangsaan Malaysia Medical Centre

Hashim Embong ( $\square$ hashimembong77@gmail.com )

Universiti Kebangsaan Malaysia Medical Centre

Shamsuriani Md Jamal

Universiti Kebangsaan Malaysia Medical Centre

Afliza Abu Bakar

Universiti Kebangsaan Malaysia Medical Centre

\section{Research Article}

Keywords: factor analysis, psychometrics, reliability, frailty

Posted Date: April 12th, 2021

DOI: https://doi.org/10.21203/rs.3.rs-385231/v1

License: (c) (i) This work is licensed under a Creative Commons Attribution 4.0 International License.

Read Full License 


\section{Abstract}

\section{Background}

The insufficiency of gold standard assessment tools is the current challenge of frailty detection in an emergency department (ED). This study aimed to translate and evaluate the psychometric properties of the Malay-translated Kihon Checklist (KCL) for frailty assessment among Malaysian elderly patients presenting to the ED.

\section{Method}

The 25-item English version of the KCL was translated to Malay language through a forward and backward translation procedure. Three expert panels considered the items and pilot-tested on 15 elderly subjects. The final version was administered to 250 elderly patients who presented in the ED. Using confirmatory factor analysis (CFA), we compared the prior factorial models of the KCL. Model fits were determined using the Chi-square test/degree of freedom ( $d f)$, comparative fit index (CFI), Tucker-Lewis index (TLI), root mean square error of approximation (RMSEA), Akaike information criterion (AIC), Bayesian information criterion (BIC), and expected cross-validation index (ECVI).

\section{Results}

Analysis revealed that none of the prior models (seven-, two-, and one-factorial model) fit the data. After modification, one-factor model with 10 items had a superior fit (Chi-square/ $d f, 54.434 / 35 ; \mathrm{CFI}, 0.962$; TLI, 0.951; RMSEA, 0.047 (95\% confidence interval (CI), 0.019-0.071); AIC, 94.434; BIC, 164.863; ECVI, 0.379). The internal consistency reliability for the pooled 10 items was acceptable, i.e., 0.786 .

\section{Conclusion}

The CFA revealed that a one-factor model with 10 items had superior goodness-of-fit than other hypothesized factorial models. The scale demonstrated adequate construct validity and acceptable reliability with caution interpretation of some items.

\section{Introduction}

Frailty is a state of heightened vulnerability resulting from an ageing-associated decline in reserve and function across multiple physiologic systems (1). There is a growing awareness for routine assessment of frailty in emergency departments (EDs) (2). Although there is no local data on the prevalence of frailty, the worldwide prevalence of frailty in EDs was reported to be $7-80 \%(3-5)$. Frail patients have been associated with multiple adverse outcomes, including disability (6), falls (7), hospitalization (8), increased healthcare cost (Kojima, 2019), poor quality of life (9), and mortality (8). The added benefit of recognizing frailty in the ED is to prevent unnecessary invasive clinical management, inform a prognosis, and plan for discharge strategies (10). 
Although multiple frailty screening tools have been developed, there is no standardization in the measurement of frailty in the ED. The two objective approaches commonly used in recognizing frailty are the phenotype model and the accumulated deficit model. The phenotype model defines frailty as a distinct clinical syndrome based on the presence of three out of the following five phenotypic criteria: unintentional weight loss, self-reported exhaustion, weakness, low physical activity, and slow walking speed (11). Meanwhile, the accumulated deficit model views frailty in the form of a frailty index. The index represents the health deficits of an individual to the total number of health deficits evaluated (12). While the phenotype model has a lack of agreed criteria for measuring each phenotype, the accumulated deficit model is too cumbersome to administer in clinical settings.

In busy EDs, self-reported frailty assessment or observed frailty is maybe more practical than objective assessment. Several instruments have been proposed for practical use in the ED, such Clinical Frailty Scale (CFS), PRISMA-7, Identification of Seniors at Risk, and Silver Code (5). The CFS, a judgment-based

frailty assessment tool, was found to be more appropriate for use in the ED environment given the speed and ease of administration (10). However, the CFS has been criticized for making inappropriate escalation treatment decisions especially in patients with a long-term stable disability (13). The challenges are to identify reliable and validated tool for safe use in the ED. A screening tool intended for use in the ED setting should be based on a simple test, should require a little time, and can be interpreted by nonspecialist professionals.

A self-reported Kihon Checklist $(\mathrm{KCL})$ has been proven to be effective in identifying and screening frail elderly patients in clinical settings (14). The KCL was developed by the Japan Ministry of Health, Labour and Welfare; it assesses seven domains of frailty: the activity of daily living, physical strength, nutrition, oral condition, socialization, memory, and mood (15). It consists of 25 items, and each item is rated either yes (1) or no (0), with a higher score indicating worse functioning (16). Although the KCL has never been validated in EDs, previous studies suggested that the instrument was easy, simple, and sensitive in identifying and screening frail elderly patients $(14,17)$. In this study, we translated the KCL into Malay and assessed the factorial validity and internal consistency in a sample of elderly patients in Malaysia ED.

\section{Materials And Methods}

The study was conducted at the ED, Universiti Kebangsaan Malaysia Medical Centre (UKMMC) in Kuala Lumpur, Malaysia, between June 2020 and December 2020. The study was conducted in three phases. Phase one includes the translation and cultural adaptation of the English version of the KCL. In phase two, content validation and pilot testing were performed. In phase three, we performed field testing to determine the reliability and factor structure of the Malay-translated KCL (Fig. 1). The study received ethical approval from the UKMMC research ethic committee (FF-2020-349).

\section{Phase one: translation process}


The permission to translate the English version of KCL to the Malay language was obtained from the original publisher. Two independent certified professional translators performed a forward translation of the English version of KCL to the Malay language; it was followed by backward translation by two different independent translators. The consensus was made on the translation of words, phrases, and items of the Malay version between researchers and translators; the Malay version was then compared with the original KCL. After some slight revisions, the final version of the Malay-translated KCL was prepared and ready to undergo the validation process.

\section{Phase two: content validation and pilot testing}

The content validity of the tool was conducted based on the quantitative methods described by Lynn (18). Three expert panels, which consist of a geriatrician, emergency physician, and family medicine specialist with an area of interest in geriatric medicine, were invited to review the Malay-translated KCL. The panels rated each item according to a four-point rating scale: 1 = not relevant, $2=$ item needs major revision, 3 = relevant but needs minor revision, and 4 = very relevant; comments that carefully assessed and reworded.

As noted by Lynn (18), the content validity index (CVI) score was estimated both at the item level (I-CVI) and scale level (S-CVI). The I-CVI was calculated by dividing the number of experts that rated an acceptable grade (rating 3 or 4 ) by the total number of expert panels. The S-CVI was calculated based on two different methods: the average method (S-CVI/Ave) and the universal agreement method (S-CVI/UA). The S-CVI/Ave is the average of the I-CVI score for all items on the scale. Meanwhile, the S-CVI/UA is the proportion of the relevant items on the scale (rating 3 or 4 ) by all experts. Based on standard recommendations for content analysis, the item with $\mathrm{I}-\mathrm{CVI}>0.79$ and scale with $\mathrm{S}-\mathrm{CVI} \geq 0.9$ was considered to have excellent content validity (19). Additionally, a modified Kappa statistic $(K)$ was computed to adjust I-CVI for chance agreement using the formula $K=(\mathrm{CVI}-P C) /(1-P C)(20)$. The probability of chance occurrence $(P C)$ was computed using the formula $P C=[N ! / A !(N-A) !] 0.5 \mathrm{~N}$, where $N$ is the number of experts, $A$ is the number agreeing on good relevance, and ! is a mathematical symbol for the product of all positive interfere less than or equal to $N$. The $K$ of 0.75 and above is considered to have the excellent agreement of relevance (21).

To confirm for face validity, we conducted a qualitative approach using cognitive interviews $(22,23)$. A pilot study was performed, in which the instrument was administered to 15 elderly patients who presented in the ED. The respondents provided feedback if they have difficulties answering the items and if any of the items were confusing or containing difficult vocabulary. The feedback was carefully assessed and reworded to give the final version of the Malay-translated KCL.

\section{Phase three: field testing}

Over 4 months between September 2020 and December 2020, a convenient sample of elderly patients aged 60 and above who presented in the ED of UKMMC were invited for the recruitment of the subject. Patients must be able to read and speak in the Malay language. We excluded patients with preexisting cognitive impairment and underlying psychiatric illness and who are critically ill. All included subjects provided written consent. Recruitment was conducted without interfering with clinical evaluation, 
investigations, treatments, and interventions by the attending clinician. The following data were documented: age, sex, race, education level, marital status, living support, comorbidity, diagnosis, and disposition.

\section{Frailty assessment}

The Malay-translated KCL was self-administered by the patients or surrogates for patients with physical difficulties. No time limit was given during the administration. In addition to the Malay KCL, we used the CFS, a clinical judgment-based screening tool that evaluates specific frailty domains including comorbidity, function, and cognition, to assess frailty. The CFS has been validated and proven to be reliable to identify frailty in EDs (24). The CFS has a total score of 9, ranging from 1 (very fit) to 9 (terminally ill), and a total score of 5 or more is considered frail (25). Measurements using either instrument represent trait or baseline at two weeks before assessment as suggested from the previous study (26).

\section{Data analysis and sample size}

Statistical analysis was performed using the IBM Statistical Package for Social Sciences (SPSS) Statistics for Windows software and SPSS Amos (version 26.0; IBM Corp., Armonk, NY, USA). A descriptive analysis of study variables was summarized using frequency and percentages for categorical variables and mean \pm SD or median (interquartile range) for continuous variables.

Confirmatory factor analysis (CFA) was performed to evaluate the factor structure of the Malay KCL. Three prior hypothesized factorial models of KCL were chosen. The first model was a seven-correlated factor model based on the original KCL (15), and the dimensions are as follows: the activity of daily living (items 1-5), physical strength (items 6-10), nutrition (items 11 and 12), oral condition (items 13-15), socialization (items 16 and 17), memory (items 18-20), and mood/cognition (items 21-25). The second model was a two-correlated factor structure based on the approach adopted by Fukutomi et al. (27). In this model, items 1-20 were classified under the lifestyle domain, and items 21-25 were classified under the mood or depression domain. The third factor was the one-factor structure where all the KCL items load on a single factor of frailty as proposed by Satake et al. (14). Model fit for each structure was assessed using the following fit statistics: $\chi 2$ goodness-of-fit test, comparative fit index (CFI), TuckerLewis Index (TLI), and root mean square error of approximation (RMSEA). The insignificant ( $p$-value > 0.05 ) model $\chi 2$ goodness-of-fit test indicates model fit. The $\chi 2$ goodness-of-fit is sensitive to the sample size. Therefore, for the well-fitting model, other fit indices, namely, CFI, TLI, and RMSEA, must be $\geq 0.93$, $\geq 0.92$, and $\leq 0.08$, respectively (28). The models were compared by examining the Akaike information criterion (AIC), Bayesian information criterion (BIC), and expected cross-validation index (ECVI), where smaller values indicated a better fit $(29,30)$.

The correlation of the KCL scores with the CFS was determined using Pearson, $r$ correlation analysis. Internal consistency was evaluated using the Cronbach's alpha with values $a>0.7$ considered acceptable reliability (31). For all analysis, $p<0.05$ was considered significant. 
In performing factor analysis, we determined the sample size according to the number of items, whereby five subjects are required per item of the questionnaire (32). With 25 items in the questionnaire, the minimum subjects were 225 .

\section{Results}

\section{Translation and cross-cultural adaptation}

The content validity of the Malay-translated KCL showed a high level of panel's agreement (Table 1). The S-CVI based on the average method and the universal agreement method were 0.97 and 0.92 , respectively, indicating excellent internal validity. Individual item was marked as relevant $(K \geq 0.75)$, except for two items, namely, numbers 14 and 19 , which were revised following a recommendation by the panels. The translated item 14 "Have you recently choked on your tea or soup?" was reworded as "tea" or "soup" is not a commonly used drink among Malaysian. The final version explained the Malay translation of "Have you recently choked on a drink?" Meanwhile, the translated item 19 "Do you make a phone call by finding the phone number?" appeared to be a functional rather than memory assessment. The item was reformulated to explain the original English version "Do you make a call by looking up phone numbers and call on your own?" After the pretest $(n=15)$, there were no changes made to the response scale. All items were well understood by the respondents. The respondents took 5-10 minutes to complete the tool. Therefore, the final version of Malay-translated KCL was accomplished and ready for field testing. 
Table 1

Calculation of I-CVI and Kappa score for the relevancy of each item $(n=3)$.

\begin{tabular}{|c|c|c|c|c|c|}
\hline Item & $\begin{array}{l}\text { Panels' } \\
\text { agreement }^{a}\end{array}$ & $\stackrel{+}{\mathrm{CV}}$ & $\mathrm{Pc}^{\mathrm{b}}$ & $K^{c}$ & Interpretation \\
\hline $\begin{array}{l}\text { Q1. Adakah anda menggunakan kenderaan } \\
\text { awam seperti bas, teksi atau grab seorang } \\
\text { diri? }\end{array}$ & 3 & 1.00 & 0.125 & 1.00 & Excellent \\
\hline \multicolumn{6}{|l|}{ Do you go out by bus or train by yourself? } \\
\hline $\begin{array}{l}\text { Q2. Adakah anda boleh keluar dan } \\
\text { menguruskan sendiri keperluan harian anda? }\end{array}$ & 3 & 1.00 & 0.125 & 1.00 & Excellent \\
\hline \multicolumn{6}{|l|}{$\begin{array}{l}\text { Do you go shopping to buy daily necessities } \\
\text { by yourself? }\end{array}$} \\
\hline $\begin{array}{l}\text { Q3. Adakah anda mengendalikan sendiri } \\
\text { deposit dan simpanan anda di bank? }\end{array}$ & 3 & 1.00 & 0.125 & 1.00 & Excellent \\
\hline \multicolumn{6}{|l|}{$\begin{array}{l}\text { Do you manage your own deposits and } \\
\text { savings at the bank? }\end{array}$} \\
\hline $\begin{array}{l}\text { Q4. Adakah anda kadang kala menziarahi } \\
\text { kawan-kawan anda? }\end{array}$ & 3 & 1.00 & 0.125 & 1.00 & Excellent \\
\hline \multicolumn{6}{|l|}{ Do you sometimes visit your friends? } \\
\hline $\begin{array}{l}\text { Q5. Adakah anda meminta pendapat dari } \\
\text { keluarga atau kawan-kawan? }\end{array}$ & 3 & 1.00 & 0.125 & 1.00 & Excellent \\
\hline \multicolumn{6}{|l|}{$\begin{array}{l}\text { Do you turn to your family or friends for } \\
\text { advice? }\end{array}$} \\
\hline $\begin{array}{l}\text { Q6. Adakah anda biasanya menaiki tangga } \\
\text { tanpa menggunakan susur tangan atau } \\
\text { dinding sebagai bantuan? }\end{array}$ & 3 & 1.00 & 0.125 & 1.00 & Excellent \\
\hline \multicolumn{6}{|l|}{$\begin{array}{l}\text { Do you normally climb stairs without using } \\
\text { handrail or wall for support? }\end{array}$} \\
\hline $\begin{array}{l}\text { Q7. Adakah anda biasanya boleh bangun } \\
\text { dari kerusi tanpa sebarang bantuan? }\end{array}$ & 3 & 1.00 & 0.125 & 1.00 & Excellent \\
\hline \multicolumn{6}{|l|}{$\begin{array}{l}\text { Do you normally stand up from a chair } \\
\text { without any aids? }\end{array}$} \\
\hline $\begin{array}{l}\text { Q8. Adakah anda biasanya boleh berjalan } \\
\text { secara berterusan selama } 15 \text { minit? }\end{array}$ & 3 & 1.00 & 0.125 & 1.00 & Excellent \\
\hline \multicolumn{6}{|l|}{$\begin{array}{l}\text { Do you normally continuously walk for } 15 \\
\text { minutes? }\end{array}$} \\
\hline $\begin{array}{l}\text { Q9. Adakah anda pernah jatuh dalam } \\
\text { tempoh masa satu tahun yang lepas? }\end{array}$ & 3 & 1.00 & 0.125 & 1.00 & Excellent \\
\hline Have you experienced a fall in the past year? & & & & & \\
\hline
\end{tabular}




\begin{tabular}{|c|c|c|c|c|c|}
\hline Item & $\begin{array}{l}\text { Panels' } \\
\text { agreement }^{\mathrm{a}}\end{array}$ & $\stackrel{+}{\mathrm{CV} I}$ & $\mathrm{Pc}^{\mathrm{b}}$ & $K^{c}$ & Interpretation \\
\hline $\begin{array}{l}\text { Q10. Adakah anda merasa bimbang yang } \\
\text { anda akan terjatuh semasa berjalan? }\end{array}$ & 3 & 1.00 & 0.125 & 1.00 & Excellent \\
\hline \multicolumn{6}{|l|}{ Do you have a fear of falling while walking? } \\
\hline $\begin{array}{l}\text { Q11. Adakah anda merasa berat badan anda } \\
\text { telah turun sebanyak } 2 \mathrm{~kg} \text { atau lebih dalam } \\
\text { masa } 6 \text { bulan ini? }\end{array}$ & 3 & 1.00 & 0.125 & 1.00 & Excellent \\
\hline \multicolumn{6}{|l|}{$\begin{array}{l}\text { Have you lost } 2 \mathrm{~kg} \text { or more in the past } 6 \\
\text { months? }\end{array}$} \\
\hline $\begin{array}{l}\text { Q12. Jika BMI kurang dari 18.5, item ini } \\
\text { ditandakan } 1\end{array}$ & 3 & 1.00 & 0.125 & 1.00 & Excellent \\
\hline \multicolumn{6}{|l|}{ If $\mathrm{BMI}$ is less than 18.5 , this item is scored 1.} \\
\hline $\begin{array}{l}\text { Q13. Adakah anda menghadapi sebarang } \\
\text { masalah untuk makan makanan yang keras } \\
\text { berbanding } 6 \text { bulan yang lalu? }\end{array}$ & 3 & 1.00 & 0.125 & 1.00 & Excellent \\
\hline \multicolumn{6}{|l|}{$\begin{array}{l}\text { Do you have any difficulties eating tough } \\
\text { foods compared to } 6 \text { months ago? }\end{array}$} \\
\hline $\begin{array}{l}\text { Q14. Adakah anda tersedak semasa minum } \\
\text { air baru-baru ini? }\end{array}$ & 2 & 0.67 & 0.375 & 0.47 & $\begin{array}{l}\text { Fair and } \\
\text { revised }\end{array}$ \\
\hline \multicolumn{6}{|l|}{$\begin{array}{l}\text { Have you recently choked on your tea or } \\
\text { soup? }\end{array}$} \\
\hline $\begin{array}{l}\text { Q15. Adakah anda selalu mengalami rasa } \\
\text { kering dalam mulut? }\end{array}$ & 3 & 1.00 & 0.125 & 1.00 & Excellent \\
\hline \multicolumn{6}{|l|}{ Do you often experience having a dry mouth? } \\
\hline $\begin{array}{l}\text { Q16. Adakah anda keluar rumah untuk } \\
\text { melakukan aktiviti sekurang-kurangnya } \\
\text { sekali seminggu? }\end{array}$ & 3 & 1.00 & 0.125 & 1.00 & Excellent \\
\hline \multicolumn{6}{|l|}{ Do you go out at least once a week? } \\
\hline $\begin{array}{l}\text { Q17. Adakah bilangan keseluruhan waktu } \\
\text { yang anda keluar dari rumah kurang pada } \\
\text { tahun ini berbanding dengan tahun lepas? }\end{array}$ & 3 & 1.00 & 0.125 & 1.00 & Excellent \\
\hline $\begin{array}{l}\text { Do you go out less frequently compared to } \\
\text { last year? }\end{array}$ & & & & & \\
\hline
\end{tabular}




\begin{tabular}{|c|c|c|c|c|c|}
\hline Item & $\begin{array}{l}\text { Panels' } \\
\text { agreement }^{a}\end{array}$ & $\stackrel{\mathrm{l}}{\mathrm{CV} I}$ & $\mathrm{Pc}^{\mathrm{b}}$ & $\mathrm{K}^{\mathrm{c}}$ & Interpretation \\
\hline $\begin{array}{l}\text { Q18. Adakah keluarga dan kawan-kawan } \\
\text { anda memberitahu anda yang anda mudah } \\
\text { lupa? }\end{array}$ & 3 & 1.00 & 0.125 & 1.00 & Excellent \\
\hline \multicolumn{6}{|l|}{$\begin{array}{l}\text { Do your family or your friends point out your } \\
\text { memory loss? }\end{array}$} \\
\hline $\begin{array}{l}\text { Q19. Adakah anda membuat panggilan } \\
\text { telefon dengan mencari nombor telefon dan } \\
\text { membuat panggilan dengan sendiri? }\end{array}$ & 2 & 0.67 & 0.375 & 0.47 & $\begin{array}{l}\text { Fair and } \\
\text { revised }\end{array}$ \\
\hline \multicolumn{6}{|l|}{$\begin{array}{l}\text { Do you make a call by looking up phone } \\
\text { numbers? }\end{array}$} \\
\hline $\begin{array}{l}\text { Q20. Adakah anda menghadapi masalah } \\
\text { mengingati tarikh hari ini? }\end{array}$ & 3 & 1.00 & 0.125 & 1.00 & Excellent \\
\hline \multicolumn{6}{|l|}{$\begin{array}{l}\text { Do you find yourself not knowing today's } \\
\text { date? }\end{array}$} \\
\hline $\begin{array}{l}\text { Q21. Dalam masa } 2 \text { minggu yang lepas, } \\
\text { adakah anda berasa kurang kepuasan } \\
\text { dalam kehidupan seharian anda? }\end{array}$ & 3 & 1.00 & 0.125 & 1.00 & Excellent \\
\hline \multicolumn{6}{|l|}{$\begin{array}{l}\text { In the last } 2 \text { weeks, have you felt a lack of } \\
\text { fulfillment in your daily life? }\end{array}$} \\
\hline $\begin{array}{l}\text { Q22. Dalam masa } 2 \text { minggu yang lepas, } \\
\text { adakah anda berasa kurang gembira } \\
\text { melakukan perkara-perkara yang dulunya } \\
\text { anda gemari? }\end{array}$ & 3 & 1.00 & 0.125 & 1.00 & Excellent \\
\hline \multicolumn{6}{|l|}{$\begin{array}{l}\text { In the last } 2 \text { weeks, have you felt lack of joy } \\
\text { when doing the things you used to enjoy? }\end{array}$} \\
\hline $\begin{array}{l}\text { Q23. Dalam masa } 2 \text { minggu yang lepas, } \\
\text { adakah anda berasa sukar untuk melakukan } \\
\text { sesuatu yang boleh anda lakukan dengan } \\
\text { mudah sebelum ini? }\end{array}$ & 3 & 1.00 & 0.125 & 1.00 & Excellent \\
\hline \multicolumn{6}{|l|}{$\begin{array}{l}\text { In the last } 2 \text { weeks, have you felt difficulty in } \\
\text { doing what you could easily do before? }\end{array}$} \\
\hline $\begin{array}{l}\text { Q24. Dalam masa } 2 \text { minggu yang lepas, } \\
\text { adakah anda berasa tidak berupaya? }\end{array}$ & 3 & 1.00 & 0.125 & 1.00 & Excellent \\
\hline \multicolumn{6}{|l|}{ In the last 2 weeks, have you felt helpless? } \\
\hline $\begin{array}{l}\text { Q25. Dalam masa } 2 \text { minggu yang lepas, } \\
\text { adakah anda berasa penat tanpa sebab? }\end{array}$ & 3 & 1.00 & 0.125 & 1.00 & Excellent \\
\hline $\begin{array}{l}\text { In the last } 2 \text { weeks, have you felt tired } \\
\text { without a reason? }\end{array}$ & & & & & \\
\hline
\end{tabular}




\begin{tabular}{|llllll}
\hline Item & $\begin{array}{l}\text { Panels' } \\
\text { agreement }^{\mathrm{a}}\end{array}$ & $\begin{array}{l}\mathrm{I}- \\
\mathrm{CVI}\end{array}$ & $\mathrm{Pc}^{\mathrm{b}}$ & $\mathrm{K}^{\mathrm{c}}$ & Interpretation \\
\end{tabular}

Abbreviations: I-CVI, item-level content validity index; ${ }^{a}$ number of panels marked 3 (relevant but needs minor revision) or 4 (very relevant); Pc, probability of chance occurrence, ${ }^{\mathrm{b}}$ calculated as [N!A!(NA)! ${ }^{*} 0.5^{\mathrm{N}} ; \mathrm{K}, \mathrm{Kappa},{ }^{\mathrm{c}}$ calculated as $\mathrm{K}=(\mathrm{I}-\mathrm{CVI}-\mathrm{Pc}) / \mathrm{1}-\mathrm{Pc}$.

\section{Subject characteristics}

The Malay KCL was tested on 250 elderly patients. The mean age of patients was $71.3 \pm 7.2$ years old, and $56 \%$ (140) were male. About half of the subjects (52.4\%) were Malay, and $13.2 \%$ had no formal education. Most of the subjects (59\%) had severe comorbidity as indicated by Charlson comorbidity score $\geq 5$. Cardiac-related conditions were the most common presentation to the $E D(n=89,35.5 \%)$, followed by surgical $(n=24,9.6 \%)$, and trauma $(n=22,8.8 \%)$.

Majority of the subjects were hospitalized $(n=165,6 \%)$. The demographic and clinical characteristics of the subjects are shown in Table 2. 
Table 2

Demographic characteristics of the subjects $(n=250)$.

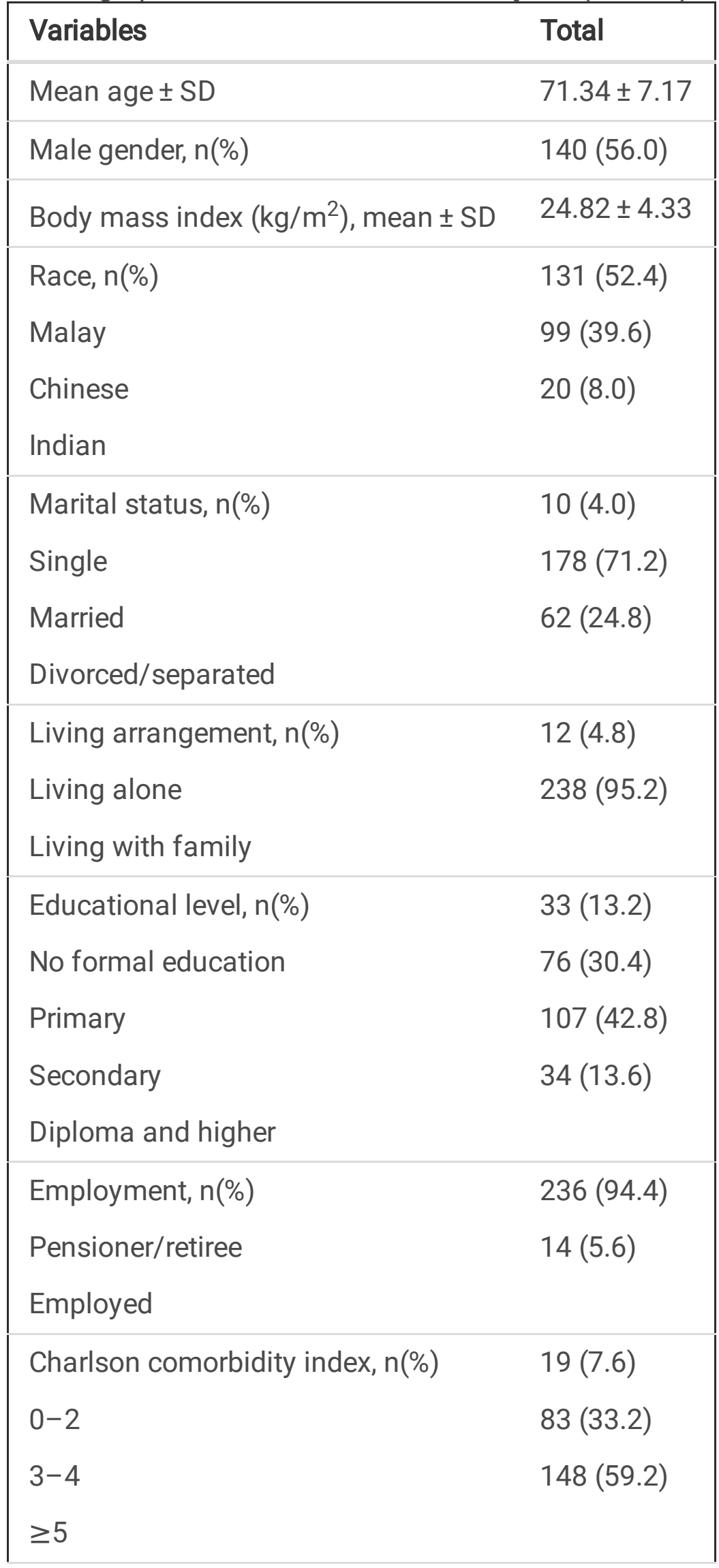




\begin{tabular}{|ll|}
\hline Variables & Total \\
\hline Clinical presentation, $\mathrm{n}(\%)$ & $89(35.6)$ \\
Cardiac & $24(9.6)$ \\
Surgical & $22(8.8)$ \\
Trauma & $17(6.8)$ \\
Respiratory & $17(6.8)$ \\
Infectious disease & $13(5.2)$ \\
Endocrine & $13(5.2)$ \\
Neurology & $13(5.2)$ \\
Musculoskeletal & $10(4.0)$ \\
Gastrointestinal & $10(4.0)$ \\
Nephrology/urology & $6(2.4)$ \\
Oncology & $16(6.4)$ \\
Others & \\
\hline Disposition, $n(\%)$ & $165(66.0)$ \\
Admitted & $85(34.0)$ \\
Discharged & \\
\hline
\end{tabular}

\section{Preliminary analysis}

Of the 250 cases, there were no missing values. Table 3 demonstrates the floor effects, ceiling effects, skewness, and kurtosis scores for the total and subdomain KCL. The skewness and kurtosis were within -2 and +2 , except for subdomain nutrition. Screening for the individual items at the univariate level revealed non-normal distribution for all items. An assessment at multivariate normality revealed the kurtosis coefficient to be 187.470 with a critical ratio of 40.337 . As the data were not normally distributed, the ML estimation with bootstrapping (33) was used to generate an accurate estimation of standard errors using the Bollen-Stine bootstrap $p$ values and confidence intervals (34). Our sample size was 250, which was adequate for bootstrapping as recommended by Nevitt and Hancock (35). A cutoff $p>0.05$ was considered for model fit. 
Table 3

Descriptive statistics for Malay-translated KCL total and subdomain scores $(n=250)$.

\begin{tabular}{|llllllll|}
\hline & $\begin{array}{l}\text { Mean } \\
(\text { SD) }\end{array}$ & Minimum & Maximum & $\begin{array}{l}\text { Reaching } \\
\text { floor (\%) }\end{array}$ & $\begin{array}{l}\text { Reaching } \\
\text { ceiling (\%) }\end{array}$ & Skew & Kurtosis \\
\hline $\begin{array}{l}\text { Total score }(25 \\
\text { items) }\end{array}$ & $\begin{array}{l}8.90 \\
(4.27)\end{array}$ & 2 & 21 & $5(2)$ & $1(0.4)$ & 0.535 & -0.327 \\
$\begin{array}{l}\text { Activity daily } \\
\text { living (5 items) }\end{array}$ & $\begin{array}{l}2.75 \\
(1.22)\end{array}$ & 0 & 5 & $4(1.6)$ & $13(5.2)$ & -0.081 & -0.940 \\
\hline $\begin{array}{l}\text { Physical } \\
\text { strength (5 }\end{array}$ & $\begin{array}{l}1.74 \\
\text { items) }\end{array}$ & 0 & 5 & $80(32.0)$ & $14(5.6)$ & 0.422 & -0.889 \\
$\begin{array}{l}\text { Nutrition (2 } \\
\text { items) }\end{array}$ & $\begin{array}{l}0.18 \\
(0.42)\end{array}$ & 0 & 2 & 207 & $3(1.2)$ & 2.127 & 3.773 \\
\hline $\begin{array}{l}\text { Oral condition } \\
\text { (3 items) }\end{array}$ & $\begin{array}{l}0.93 \\
(0.85)\end{array}$ & 0 & 3 & $89(35.6)$ & $11(4.4)$ & 0.571 & -0.432 \\
\hline $\begin{array}{l}\text { Socialization (2 } \\
\text { items) }\end{array}$ & $\begin{array}{l}1.54 \\
(0.56)\end{array}$ & 0 & 2 & $8(3.2)$ & $142(56.8)$ & -0.695 & -0.559 \\
\hline $\begin{array}{l}\text { Memory (3 } \\
\text { items) }\end{array}$ & $\begin{array}{l}0.44 \\
(0.72)\end{array}$ & 0 & 3 & $\begin{array}{l}170 \\
(68.0)\end{array}$ & $3(1.2)$ & 1.489 & 1.302 \\
\hline Mood (5 items) & $\begin{array}{l}1.32 \\
(1.36)\end{array}$ & 0 & 5 & $90(36.0)$ & $4(1.6)$ & 0.845 & -0.310 \\
\hline
\end{tabular}

\section{Factor analysis}

The three measurement models tested to evaluate the factor structure of the Malay $\mathrm{KCL}$ are shown in Fig. 2. The results showed that none of the models was a good fit for the data. All models were revised with careful observation of the modification indices. The model fit indices for seven-, two-, and one-factor models are shown in Table 4. Overall, multiple fit indices demonstrated that the best model fit was the one-factor model with 12 items (Chi-square/degree of freedom ( $d f), 81.474 / 54$ ( $p=0.096) ; \mathrm{CFI}, 0.947$; TLI, 0.935; RMSEA, 0.045 (95\% confidence interval (Cl), 0.023-0.064); AIC, 129.474; BIC, 212.989; ECVI, 0.520 ). The standardized coefficients ranged from -0.01 to 0.78 , and 10 of the 12 items were statistically significant $(p<0.05)$. These two items were removed, and the final model comprises 10 items with fit indices of Chi-square/ $d f, 54.434 / 35$ ( $p=0.116$ ); CFI, 0.962; TLI, 0.951; RMSEA, 0.047 (95\% Cl, 0.0190.071); AIC, 94.434; BIC, 164.863; and ECVI, 0.379 (Fig. 3). Scores on the three instruments that significantly correlated with each other suggested good convergent validity (Malay KCL 10 items vs. 25 items, $r=0.925$; Malay KCL 10 items vs. CFS, $r=0.787$ ). 
Table 4

Summary for model fit indices for the Malay KCL confirmatory factor models.

\begin{tabular}{|c|c|c|c|c|c|c|c|c|}
\hline Model & $\begin{array}{l}\text { Chi- } \\
\text { square/df }\end{array}$ & $\begin{array}{l}\text { Bollen- } \\
\text { Stine } \\
\text { bootstrap, } \\
p\end{array}$ & CFI & TLI & $\begin{array}{l}\text { RMSEA } \\
(95 \% \\
\mathrm{Cl})\end{array}$ & AIC & BIC & ECVI \\
\hline Model 1 & $536.818 / 524$ & 0.004 & 0.770 & 0.728 & $\begin{array}{l}0.067 \\
(0.059- \\
0.075)\end{array}$ & 678.818 & 928.842 & 2.726 \\
\hline Model 2 & $744.573 / 274$ & 0.004 & 0.596 & 0.560 & $\begin{array}{l}0.085 \\
(0.078- \\
0.092)\end{array}$ & 846.573 & 1026.167 & 3.400 \\
\hline Model 3 & $771.015 / 275$ & 0.004 & 0.617 & 0.581 & $\begin{array}{l}0.083 \\
(0.076- \\
0.090)\end{array}$ & 871.015 & 1047.088 & 3.498 \\
\hline $\begin{array}{l}\text { Model } 1 \\
\text { (revised) }\end{array}$ & $236.861 / 168$ & 0.109 & 0.922 & 0.902 & $\begin{array}{l}0.041 \\
(0.028- \\
0.052)\end{array}$ & 362.861 & 584.714 & 1.457 \\
\hline $\begin{array}{l}\text { Model } 2 \\
\text { (revised) }\end{array}$ & $104.523 / 76$ & 0.104 & 0.950 & 0.940 & $\begin{array}{l}0.039 \\
(0.017- \\
0.056)\end{array}$ & 162.523 & 264.645 & 0.653 \\
\hline $\begin{array}{l}\text { Model } 3 \\
\text { (revised) }\end{array}$ & $81.474 / 54$ & 0.096 & 0.947 & 0.935 & $\begin{array}{l}0.045 \\
(0.023- \\
0.064)\end{array}$ & 129.474 & 212.989 & 0.520 \\
\hline \multicolumn{9}{|c|}{$\begin{array}{l}\text { Model 1, seven-factor model based on Arai and Satake (2015); Model 2, two-factor model based on } \\
\text { Fukutomi et al. (2015); Model 3, one-factor model based on Satake et al. (2016). }\end{array}$} \\
\hline \multicolumn{9}{|c|}{$\begin{array}{l}d f \text {, degree of freedom; CFI, comparative fit index; TLI, Tucker-Lewis index; NFI, normed fit index; } \\
\text { RMSEA, root mean square error of approximation; Cl, confidence interval; AIC, Akaike information } \\
\text { criterion; BIC, Bayesian information criterion; ECVI, expected cross-validation index. }\end{array}$} \\
\hline
\end{tabular}

\section{Reliability}

The analysis of the reliability of the Cronbach's alpha for the pooled 10 items showed acceptable internal consistency, i.e., 0.786. Most items appeared to have a good correlation with other items (item-total correlation >0.3), except for items 5 and 13 (range, 0.187-0.647) (Table 5). 
Table 5

Item-total statistics for the one-factor model of the Malay KCL with six items ( $\mathrm{n}=$ 250).

\begin{tabular}{|lll|}
\hline Items & Corrected item-total correlation & Cronbach's alpha if item is deleted \\
\hline Q2 & 0.498 & 0.762 \\
\hline Q5 & 0.224 & 0.790 \\
\hline Q6 & 0.611 & 0.746 \\
\hline Q7 & 0.597 & 0.749 \\
\hline Q8 & 0.647 & 0.740 \\
\hline Q13 & 0.187 & 0.793 \\
\hline Q14 & 0.308 & 0.784 \\
\hline Q16 & 0.455 & 0.768 \\
\hline Q22 & 0.372 & 0.777 \\
\hline Q23 & 0.557 & 0.754 \\
\hline
\end{tabular}

\section{Discussion}

In this study, we translated the English version of the KCL into Malay and evaluated the validity and reliability in a sample of elderly patients in the ED. The psychometric evaluation showed satisfactory validity and reliability, supporting the use of the one-factor model with 10 items. In the first part of the study, we translated and evaluated the content validation of the instrument. We adopted the Lynn method (18), an accepted approach for establishing the content validity of instruments. The findings suggested a high degree of agreement beyond chance in most of the individual item as measured by the modified Kappa. A small number of items had a minor cultural and syntactical issue, and both items were revised through discussions with the research team. The instrument was pretested using cognitive interview to enhance the face validity of the instrument (36). Together with face validity, the instrument provides a robust foundation for future validation of the Malay-translated KCL.

There is limited consensus regarding the dimension of the KCL. The multidimensional factor structure of the $\mathrm{KCL}$ was proposed based on the original version, in which the items are divided across seven structured domains (15). Meanwhile, Satake et al. proposed for unidimensional factor structure based on the significant correlation between the total KCL score with frailty phenotypes (14). To date, only one study examined the factor structure of the KCL (37). The Spanish version of the KCL suggested the onefactor model with 15 items in a sample of community-dwelling Spanish older adults (37). In this study, we conducted a CFA to confirm whether the prior hypothesized factor models of the KCL were applicable to Malaysian elderly patients in ED settings. 
We did not perform an exploratory factor analysis as the CFA provides stronger evidence in support of the validity of the instrument with regard to the model dimension (38). After carrying CFA, none of the models confirmed the factor structure of the Malay KCL, comparable with the findings reported in the Spanish sample (37). The proposed one-factor model of the Malay KCL fit well after extensive revisions with many of the items were deleted. However, only six items had factor loading larger than 0.5 , which is the recommended minimum factor loading in CFA (39). Most of the remaining items predominantly relate to function and physical concern, which might reflect findings from previous studies that have suggested that the physical function remains the most recognized features of frailty in the elderly $(11,40)$. In addition, a previous study suggested that the physical component of the original KCL had the highest influence on frailty score (41). As suggested by Awang (Awang, 2015), the items were maintained as the fit statistics have achieved the required level.

The internal consistency reliability of the 10-item Malay KCL was found to be acceptable. The item-total correlation for most items was seen to be within $0.30-0.70$, indicating a good relationship of items with the construct (42). The total score for the 10-item Malay KCL was significantly correlated with the total score of the 25 -item KCL, suggesting that both versions shared a similar construct. The 10-item Malay $\mathrm{KCL}$ also showed a strong correlation with the CFS, indicating that these two tools measure similar concepts. However, because a large number of items (> 20\%) were removed during the model revision, the model needs a revalidation study on a new sample of older patients in the ED (43).

The findings of our study did not support the KCL definition of frailty as there was insufficient cognitive and nutrition evaluation in the instrument. These findings should be interpreted in the context of potential limitation. First, the current study excluded patients with an underlying cognitive impairment since they are often viewed as barriers to the completion of self-reported questionnaires. Patients with dementia may exhibit a wide range of cognitive and behavioral symptoms that potentially interfere with their ability to answer self-reported questionnaires. However, several studies emphasized the inclusion of patients with cognitive impairment in frailty assessment (44). It is also argued the nutrition component is underdeveloped. The cutoff body mass index (BMI) of $18.5 \mathrm{~kg} / \mathrm{m} 2$ to define frailty have seen conflicting evidence, in which study by Crow and colleagues has suggested of a poor relationship between frailty and BMI-defined obesity (45). Second, although KCL was self-administered, in some instances, we asked relatives as a surrogate to fill in the questionnaire. Family members might not recognize or addressed subtle changes in frailty features among patients. In some instances, caregivers might overestimate the actual functional deficits than did patients (46).

\section{Conclusion}

This study supports the acceptable psychometric properties of the Malay-translated KCL in a sample of an elderly patient in the ED. The CFA revealed that the one-factor model with 10 items had superior goodness-of-fit than other hypothesized models. The scale demonstrated adequate convergent validity and acceptable reliability with caution interpretation of some items. 


\section{Declarations}

\section{ETHIC APPROVAL AND CONSENT TO PARTICIPATE}

The study received ethical approval from the University Kebangsaan Malaysia Medical Centre (UKMMC) research ethic committee (FF-2020-349).

All included subjects provided written informed consent.

All methods were carried out in accordance with relevant guidelines and regulations.

\section{CONSENT FOR PUBLICATION}

Not applicable

\section{AVAILABILITY OF DATA AND MATERIALS}

The data generated or analysed during this study are included in this published article [Raw data Kihon.csv].

\section{COMPETING INTERESTS}

No conflict of interest has been declared by the authors.

\section{FUNDINGS}

No funding

\section{AUTHOR CONTRIBUTIONS}

$\mathrm{HE}, \mathrm{SMJ}$ and $\mathrm{AAB}$ designed the study.

SNLS collected the data.

HE and SNLS performed the analyses and interpreted the data.

HE supervised the study.

HE and SNLS drafted the manuscript.

All authors read and approved the final manuscript.

\section{ACKNOWLEDGMENTS}

The authors are grateful to the Faculty of Language and Linguistic, University of Malaya, for the translation of the instrument. We would like to thank to the expert panels that gently agreed to participate in this study: Associate Professor Dr. Mohd Izwan Zakaria, Trauma and Emergency Department, Faculty 
of Medicine, University of Malaya; Associate Professor Dr. Noorlaili Mohd Tohit, Department of Family Medicine, Faculty of Medicine, National University of Malaysia; and Dr. Hazlina Mahadzir, Department of Internal Medicine, National University of Malaysia.

\section{AUTHOR'S INFORMATION}

\section{Affiliation}

Department of emergency medicine, Faculty of Medicine, Universiti Kebangsaan Malaysia Medical Centre, Jalan Yaacob Latif, Bandar Tun Razak, 56000 Cheras, Kuala Lumpur, Malaysia

Authors: Suhaila Naema Laili Suhairi, Hashim Embong, Shamsuriani Md Jamal, Afliza Abu Bakar

Corresponding author: Hashim Embong

\section{References}

1. Xue QL. The frailty syndrome: Definition and natural history. Clinics in Geriatric Medicine. 2011;27(1):1-15.

2. Jørgensen R, Brabrand M. Screening of the frail patient in the emergency department: A systematic review. European Journal of Internal Medicine. 2017;45:71-3.

3. Joseph B, Pandit V, Zangbar B, Kulvatunyou N, Hashmi A, Green DJ, et al. Superiority of frailty over age in predicting outcomes among geriatric trauma patients: A prospective analysis. JAMA Surgery. 2014;149(8):766-72.

4. Wallis SJ, Wall J, Biram RW, Romero-Ortuno R. Association of the clinical frailty scale with hospital outcomes. QJM. 2015;108(12):943-9.

5. Elliott A, Hull L, Conroy SP. Frailty identification in the emergency department-A systematic review focussing on feasibility. Age \& Ageing. 2017;46(3):509-13.

6. Choi YS, Kim MJ, Lee GY, Seo YM, Seo AR, Kim B, et al. The association between frailty and disability among the elderly in rural areas of Korea. International Journal of Environmental Research \& Public Health. 2019;16(14).

7. Cheng $\mathrm{MH}$, Chang SF. Frailty as a risk factor for falls among community dwelling people: Evidence from a meta-analysis. Journal of Nursing Scholarship. 2017;49(5):529-36.

8. Hao Q, Zhou L, Dong B, Yang M, Dong B, Weil Y. The role of frailty in predicting mortality and readmission in older adults in acute care wards: A prospective study. Scientific Reports. 2019;9(1):1207-.

9. Henchoz Y, Büla C, Guessous I, Santos-Eggimann B. Association between physical frailty and quality of life in a representative sample of community-dwelling Swiss older people. Journal of Nutrition, Health \& Aging. 2017;21(5):585-92. 
10. Lewis ET, Dent E, Alkhouri H, Kellett J, Williamson M, Asha S, et al. Which frailty scale for patients admitted via Emergency Department? A cohort study. Archives of Gerontology \& Geriatrics. 2019;80:104-14.

11. Fried LP, Tangen CM, Walston J, Newman AB, Hirsch C, Gottdiener J, et al. Frailty in older adults: Evidence for a phenotype. The Journals of Gerontology. Series A, Biological Sciences \& Medical Sciences. 2001;56(3):M146-56.

12. Mitnitski AB, Mogilner AJ, Rockwood K. Accumulation of deficits as a proxy measure of aging. The Scientific World JOURNAL. 2001;1:323-36.

13. Sablerolles RSG, Lafeber M, van Kempen JAL, van de Loo BPA, Boersma E, Rietdijk WJR, et al. Association between Clinical Frailty Scale score and hospital mortality in adult patients with COVID19 (Comet): An international, multicentre, retrospective, observational cohort study. The Lancet. Healthy Longevity. 2021;2(3):e163-70.

14. Satake S, Senda K, Hong YJ, Miura H, Endo H, Sakurai T, et al. Validity of the K ihon Checklist for assessing frailty status. Geriatrics \& Gerontology International. 2016;16(6):709-15.

15. Arai H, Satake S. English translation of the Kihon Checklist. Geriatrics \& Gerontology International. 2015;15(4):518-9.

16. Sewo Sampaio PY, Sampaio RA, Yamada M, Arai H. Systematic review of the Kihon Checklist: Is it a reliable assessment of frailty? Geriatrics \& Gerontology International. 2016;16(8):893-902.

17. Esenkaya ME, Dokuzlar O, Soysal P, Smith L, Jackson SE, Isik AT. Validity of the Kihon Checklist for evaluating frailty status in Turkish older adults. Geriatrics \& Gerontology International. 2019;19(7):616-21.

18. Lynn MR. Determination and quantification of content validity. Nursing Research. 1986;35(6):382-5.

19. Zamanzadeh V, Ghahramanian A, Rassouli M, Abbaszadeh A, Alavi-Majd H, Nikanfar AR. Design and Implementation Content Validity Study: Development of an instrument for measuring PatientCentered Communication. Journal of Caring Sciences. 2015;4(2):165-78.

20. Polit DF, Beck CT, Owen SV. Is the CVI an acceptable indicator of content validity? Appraisal and recommendations. Research in Nursing \& Health. 2007;30(4):459-67.

21. Cicchetti DV, Sparrow SA. Developing criteria for establishing interrater reliability of specific items: Applications to assessment of adaptive behavior. American Journal of Mental Deficiency. 1981;86(2):127-37.

22. Collins D. Pretesting survey instruments: An overview of cognitive methods. Quality of Life Research. 2003;12(3):229-38.

23. Willis GB, Miller K. Cross-cultural cognitive interviewing: Seeking comparability and enhancing understanding. Field Methods. 2011;23(4):331-41.

24. Wallis SJ, Wall J, Biram RWS, Romero-Ortuno R. Association of the clinical frailty scale with hospital outcomes. QJM. 2015;108(12):943-9. 
25. Church S, Rogers E, Rockwood K, Theou O. A scoping review of the Clinical Frailty Scale. BMC Geriatrics. 2020;20(1):393.

26. Jarrett PG, Rockwood K, Carver D, Stolee P, Cosway S. Illness presentation in elderly patients. Archives of Internal Medicine. 1995;155(10):1060-4.

27. Fukutomi E, Okumiya K, Wada T, Sakamoto R, Ishimoto Y, Kimura Y, et al. Relationships between each category of 25-item frailty risk assessment (Kihon Checklist) and newly certified older adults under Long-Term Care Insurance: A 24-month follow-up study in a rural community in Japan. Geriatrics \& Gerontology International. 2015;15(7):864-71.

28. Bagozzi RP, Yi Y. Specification, evaluation, and interpretation of structural equation models. Journal of the Academy of Marketing Science. 2012;40(1):8-34.

29. Burnham KP, Anderson DR. Multimodel inference: Understanding AIC and BIC in model selection. Sociological Methods \& Research. 2004;33(2):261-304.

30. Schreiber JB, Nora A, Stage FK, Barlow EA, King J. Reporting structural equation modeling and confirmatory factor analysis results: A review. The Journal of Educational Research. 2006;99(6):32338.

31. Cronbach LJ. Coefficient alpha and the internal structure of tests. Psychometrika. 1951;16(3):297334.

32. DeVon HA, Block ME, Moyle-Wright P, Ernst DM, Hayden SJ, Lazzara DJ, et al. A psychometric toolbox for testing validity and reliability. Journal of Nursing Scholarship. 2007;39(2):155-64.

33. Byrne BM. Structural equation modeling with Mplus: Basic concepts, applications, and programming: Routledge; 2013.

34. Bollen KA, Stine RA. Bootstrapping goodness-of-fit measures in structural equation models. Sociological Methods \& Research. 1992;21(2):205-29.

35. Nevitt J, Hancock GR. Performance of bootstrapping approaches to model test statistics and parameter standard error estimation in structural equation modeling. Structural Equation Modeling: A Multidisciplinary Journal. 2001;8(3):353-77.

36. Egger-Rainer A. Enhancing validity through cognitive interviewing. A methodological example using the Epilepsy Monitoring Unit Comfort Questionnaire. Journal of Advanced Nursing. 2019;75(1):22433.

37. Sentandreu-Mañó T, Fernández I, Cebrià I Iranzo MÀ, Tomás JM, Cebria i Iranzo MA. Dimensions underlying frailty indicators in the Kihon Checklist. Geriatrics \& Gerontology International. 2019;19(10):982-7.

38. Brown TA. Confirmatory factor analysis for applied research: Guilford Publications; 2015.

39. Chin WW. The partial least squares approach to structural equation modeling. Modern Methods for Business Research. 1998;295(2):295-336.

40. Gale CR, Cooper C, Aihie Sayer A. Prevalence of frailty and disability: Findings from the English Longitudinal Study of Ageing. Age \& Ageing. 2014;44(1):162-5. 
41. Sewo Sampaio PY, Sampaio RA, Yamada M, Ogita M, Arai H. Validation and translation of the Kihon Checklist (frailty index) into Brazilian Portuguese. Geriatrics \& Gerontology International. 2014;14(3):561-9.

42. De Vaus D, de Vaus D. Surveys in social research: Routledge; 2013.

43. Hair JF. Multivariate data analysis; 2009.

44. Sternberg SA, Wershof Schwartz AW, Karunananthan S, Bergman H, Mark Clarfield A. The identification of frailty: A systematic literature review. Journal of the American Geriatrics Society. 2011;59(11):2129-38.

45. Crow RS, Lohman MC, Titus AJ, Cook SB, Bruce ML, Mackenzie TA, et al. Association of Obesity and Frailty in older adults: NHANES 1999-2004. The Journal of Nutrition, Health \& Aging. 2019;23(2):13844.

46. Magaziner J, Zimmerman SI, Gruber-Baldini AL, Hebel JR, Fox KM. Proxy reporting in five areas of functional status: Comparison with self-reports and observations of performance. American Journal of Epidemiology. 1997;146(5):418-28.

\section{Figures}




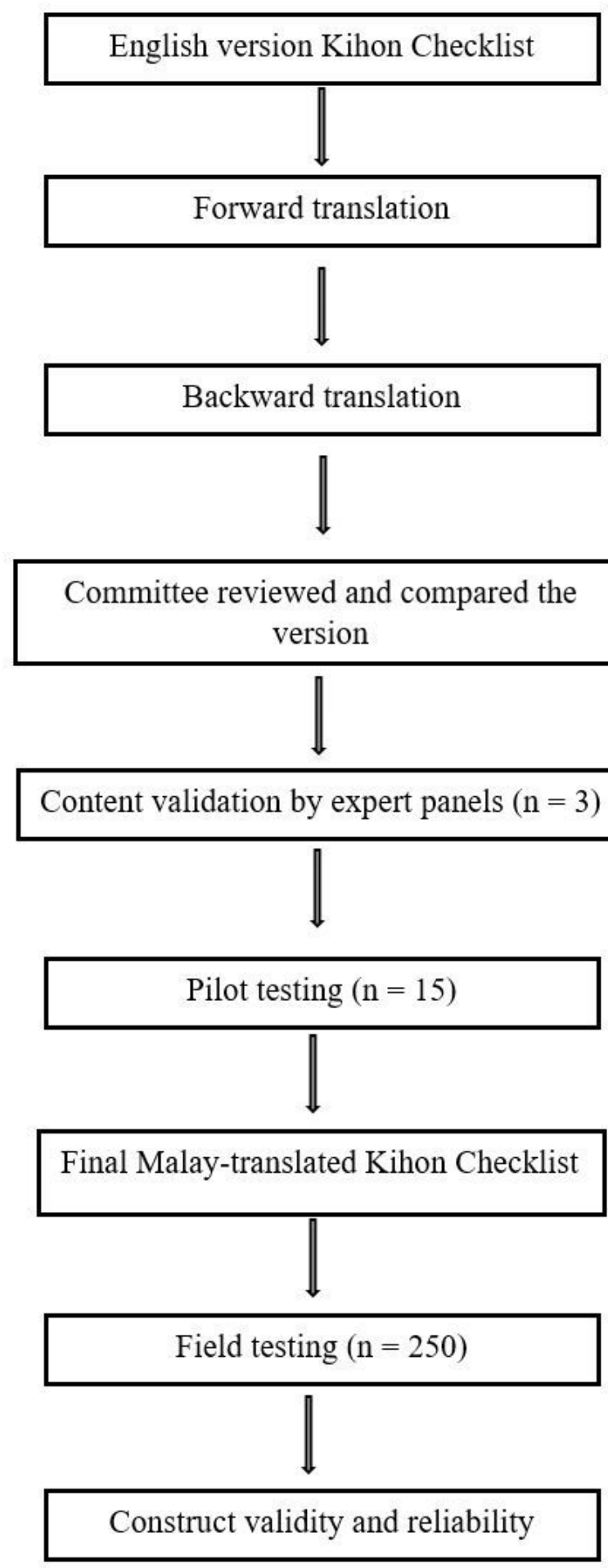

\section{Figure 1}

Flowchart of the translation process and cross-cultural adaptation. 


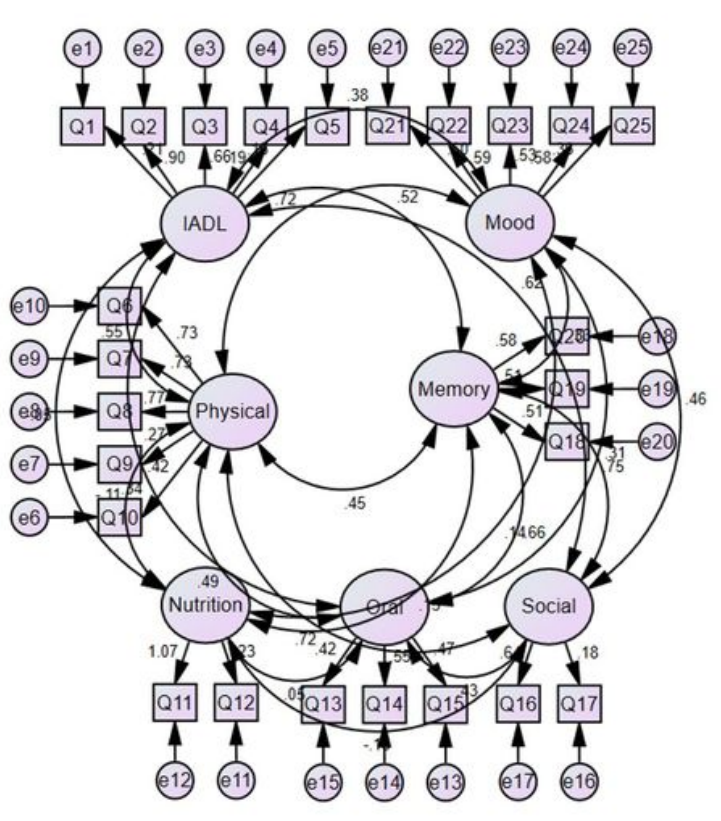

Model 1

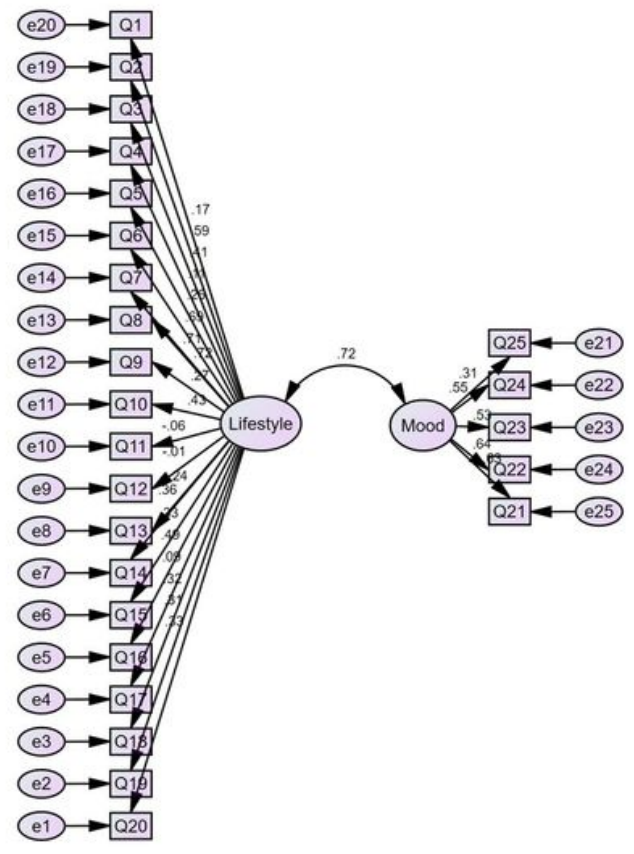

Model 2

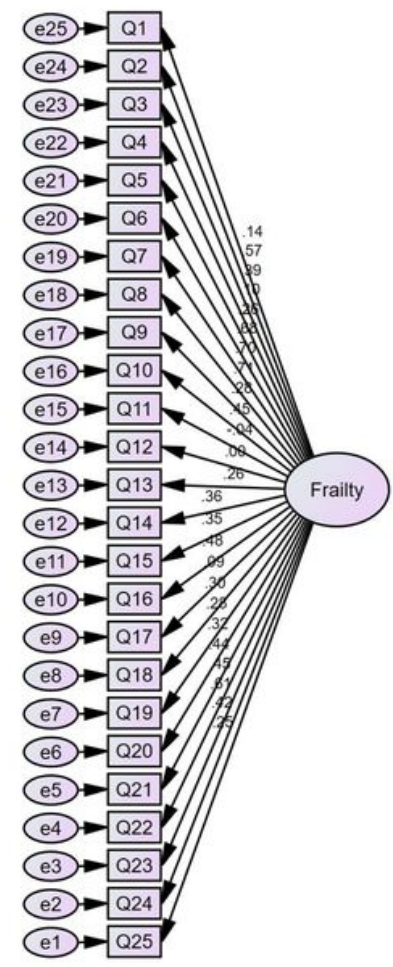

Model 3

Figure 2

Path diagram of the factor structure of the Malay-translated KCL. Model 1: Seven-factor model based on Satake et al. Model 2: Two-factor model based on Fukutomi et al. Model 3: One-factor model with 25 items based on Satake et al. 


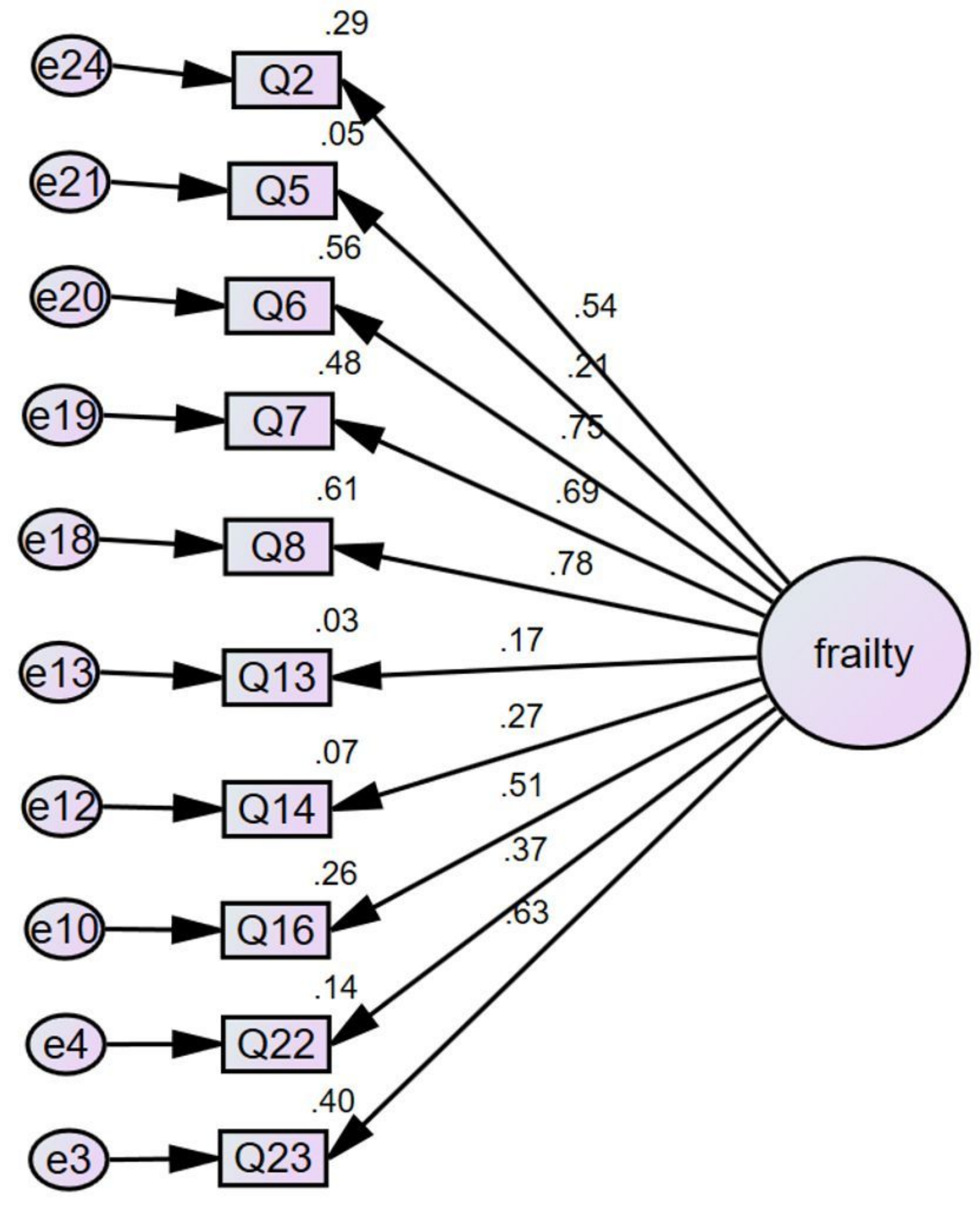

Figure 3

The revised one-factor model of the Malay KCL with 10 items.

\section{Supplementary Files}

This is a list of supplementary files associated with this preprint. Click to download. 
- RawdataKihon.csv

Page 25/25 